\title{
Differential effects of intervention timing on COVID-19 spread in the United States
}

\author{
Sen Pei, Sasikiran Kandula and Jeffrey Shaman* \\ Department of Environmental Health Sciences, Mailman School of Public Health, Columbia University, New York, NY 10032, USA. \\ *Corresponding author. Email: jls106@cumc.columbia.edu
}

Assessing the effects of early non-pharmaceutical interventions on COVID-19 spread is crucial for understanding and planning future control measures to combat the pandemic. We use observations of reported infections and deaths, human mobility data, and a metapopulation transmission model to quantify changes in disease transmission rates in US counties from March 15 to May 3, 2020. We find that marked, asynchronous reductions of the basic reproductive number occurred throughout the US in association with social distancing and other control measures. Counterfactual simulations indicate that, had these same measures been implemented 1-2 weeks earlier, substantial cases and deaths could have been averted, and that delayed responses to future increased incidence will facilitate a stronger rebound of infections and death. Our findings underscore the importance of early intervention and aggressive control in combatting the COVID-19 pandemic.

\section{Introduction}

The ongoing COVID-19 pandemic has caused millions of infections and hundreds of thousands of deaths worldwide (1, 2 ). In the United States, the first imported case of COVID-19 was reported on January 20, 2020 (3). In subsequent weeks, community transmission was established, and the causative pathogen, SARS-CoV-2, quickly spread throughout the entire country (2). As of June 22, 2020, over 2.3 million infections and 120 thousand deaths had been confirmed nationwide, making the US the hardest-hit country in the world to date (4).

In an effort to slow the spread of COVID-19, control measures enforcing social distancing and restricting individual contact were implemented across the US beginning in mid-March. In other countries, these non-pharmaceutical interventions (NPIs) have successfully limited the spread of COVID-19 (5-12); however, in the US, the effectiveness of these control measures has been less pronounced. It is therefore important that changes in virus transmissibility within the US, due to NPIs, be quantified, so that the effects of earlier interventions on cases and deaths can be evaluated.

\section{Results}

Inference ofCOVID-19 transmission dynamics in the US In this study we adapted and applied a dynamic metapopulation model $(13,14)$ informed by human mobility data (15, 16) (Fig. S1) and representing SARS-CoV-2 transmission in 3142 US counties (see Materials and Methods). During the study period, February 21, 2020 to May 3, 2020, measures to control the virus were actively, but heterogeneously, implemented throughout the US. We explicitly simulated documented and undocumented infections (17), for which separate transmission rates, $\beta$ and $\mu \beta(\mu<1)$, respectively, are defined. Here $\mu$ is the relative transmissibility of undocumented infections. To reflect heterogeneity in transmission rates across the US while avoiding a large number of model parameters, we defined a separate $\beta_{i}$ for counties with greater than 400 cumulative confirmed cases as of May 3, 2020 ( $n=311$ ) (Fig. S1). The remaining 2831 counties were apportioned among 16 additional transmission rate parameters depending on cumulative case levels and population density (see Materials and Methods). Other parameters in the model include the ascertainment rate, $\alpha$, which represents the fraction of infections documented as confirmed cases, the average latency period, $Z$, the average duration of infectiousness, $D$, and a travel multiplicative factor, $\theta$.

The metapopulation model explicitly simulates intercounty mobility using observed rates of inter-county visits to points of interest (POI) (e.g., restaurants, stores, etc.) on a county-by-county basis. Intra-county mobility is not represented as the relationship between mobility and disease transmission is unknown. Instead, we inferred changing transmission rates within counties using from time series records of COVID-19 activity. This parameter estimation was performed using the ensemble adjustment Kalman filter (EAKF) (18) in conjunction with county-level observations of both daily reported cases and deaths in the US (19) (see Materials and Methods, Supplementary Materials). We focus on the national fitting, as well as major metropolitan areas with large populations and abundant data, for which parameter estimates are well informed. Further, as many states 
reopened portions of their economies in early May, the study period was limited to February 21, 2020 - May 3, 2020 when active control efforts were in place.

Daily cases and deaths in the US and the New York metropolitan area are well fit by the transmission model (Fig. 1AD). Model estimates for counties with large number of cases and deaths (Fig. S2) yield low discrepancy from observations (Table S1). The inferred basic reproductive numbers, $R_{t} \equiv \beta D[\alpha+(1-\alpha) \mu](17,20)$, for six metropolitan areas New York, New Orleans, Los Angeles, Chicago, Boston and Miami - on five dates (March 15, March 29, April 12, April 26, May 3) are shown in Table 1 (see Materials and Methods). After March 15, $R_{t}$ in all six metropolitan areas decreases substantially in association with the implementation of social-distancing policies and practices (Fig. S3). The estimated effective reproductive numbers, $R_{e} \equiv \beta D[\alpha+(1-\alpha) \mu] S / N$, for these six metropolitan areas also decrease after March 15 , 2020 (Fig. 1E). In three of the six metropolitan areas $R_{e}$ is well below 1 as of May 3, 2020. For Chicago, Los Angeles and Miami, where daily confirmed cases and deaths were still increasing or becoming stable (Fig. S1), $R_{e}$ is close to 1 . In the New York metropolitan area $R_{e}$ dropped below 1 on April 4 and continued decreasing since then. The estimated nationwide ascertainment rate declined from 0.20 around March 16, a time of rapid COVID-19 spread and then stabilized around 0.1 after April 5 (Fig. 1F). Note that this finding indicates that, prior to April 5, even though testing capacity had increased substantially, daily new infections increased faster, yielding a declining ascertainment rate.

Estimated cumulative infections (both reported and unreported) for the New York metropolitan area on March 15, 2020 are one order of magnitude higher than for the other five metropolitan areas (Fig. 1G). Thus, although the estimated $R_{e}$ in New York from March 15 to May 3, 2020 was comparable to or lower than these other areas, the attack rate in New York remains roughly an order of magnitude higher through May 3, 2020. We also overlaid the inferred $R_{t}$ for the six metropolitan areas with the dates on which local social distancing orders were announced (21) (Fig. S3). In general, $R_{t}$ decreases as more interventions are implemented; however, there are no abrupt changes of $R_{t}$ associated with the timing of the local interventions, possibly due to a more gradual adjustment of individual human behaviors. The estimated effective reproductive numbers on five dates (March 15, March 29, April 12, April 26 and May 3) for 311 counties with cumulative cases $>=400$ until May 3 are available online (22). Sensitivity analysis indicates that the inference results are robust to the duration of daytime and nighttime transmission imposed in the model (Fig. S4).

\section{Asynchronous transmission reduction in the US}

We observe an asynchronous reduction of $R_{e}$ among counties with 400 or more cases by May 3, and across all 3142 counties the estimated effective reproductive numbers exhibit considerable variability (Fig. S5). To visualize the speed at which local transmission rates were reduced, we show the first dates when $R_{e}$ dropped below certain threshold values $(1.5,1.25,1$ and 0.75) and stayed below those thresholds until May 3 (Fig. 2). Some metropolitan areas such as San Francisco, New York City and New Orleans reduced $R_{e}$ below one during April and kept it below one through May 3. Less populous counties in the mountain region of the US also have low effective reproductive numbers, possibly due to lower population density supporting fewer opportunities for sustained transmission. At the same time, a large number of counties still have $R_{e}$ above one as of May 3, indicating that local transmission had not yet been effectively curbed. The asynchronous reduction of transmission rates, partly due to different timelines of local control orders, differing compliance to social distancing rules, and differing starting values of $R_{e}$, complicates containment and control of COVID-19. Locations with sustained transmission can re-introduce infections to locations where transmission is well suppressed, once control measures are relaxed or lifted, possibly increasing opportunities for local transmission and case growth.

The model-inference system enables estimation of the evolution of susceptibility in county populations during the pandemic. Nationwide, $95.4 \%$ (93.8\%-96.6\%) of the US population remained susceptible as of May 3, with notable differences in key metropolitan areas (Fig. S6). Specifically, the estimated susceptible population percentage in the New York metropolitan area was $75.5 \%$ (70.5\%-79.4\%), which roughly agrees with the $21 \%$ seroprevalence reported for New York City on April 23 (23). Further, the estimated cumulative percentage of infected individuals also generally agrees with the reported seroprevalence rates obtained from an independent large-scale serological study $(24,25)$ for a variety of locations and dates (Fig. 1H). On May 3, even counties with a large number of confirmed cases still had high population susceptibility, revealing an absence of herd immunity and continued risk of additional COVID-19 waves. The estimated susceptible population on May 3 in the 100 counties with the most reported cases is available at (22). Additional validations of the estimates of $R_{t}$ and initial prevalence in the US against other independent studies are provided in Materials and Methods.

\section{Counterfactual simulations of COVID-19 spread}

The inference results indicate that the NPIs varyingly adopted in the US after March 15 reduced rates of COVID-19 
transmission. During the initial growth of a pandemic, infections increase exponentially. As a consequence, early intervention and rapid response are critical for limiting morbidity and mortality. To quantify the effects of earlier interventions on COVID-19 outcomes in the US, we performed two counterfactual simulations in which the sequence of transmission rates and ascertainment rate inferred for March 15 - May 3, 2020, were shifted back 1 and 2 weeks, i.e., to March 8, 2020 and March 1, 2020, respectively. Specifically, we ran the inference from February 21 to March 8 or March 1 to constrain the initial model state, and then applied the daily posterior parameters, i.e., $\alpha$ and $\beta$ s, as estimated beginning March 15. The simulations were generated until May 3, 2020. For the last 1-2 weeks without inferred parameters due to the shift in the time window, we applied the final parameter estimates of May 3, 2020, the last day of inference. This approach shifts the asynchronous control timelines for different counties back 1 or 2 weeks, something a local or aggregate transmission model representing a single geography cannot represent. Further, the metapopulation model construct enables incorporation of the inter-county dynamical interaction of disease transmission, which is crucial for the spatial expansion of COVID-19 during the early stage of the pandemic.

The counterfactual simulations indicate that had observed control measures been adopted one week earlier, the US would have avoided 601,667 (95\% CI: 464,381- 722,880) [52.6\% (40.6\%-63.1\%)] confirmed cases and 32,335 (23,60040,573) [49.4\% (36.1\%-62.0\%)] deaths nationwide as of May 3, 2020 (Fig. 3A-B). In the New York metropolitan area, the epicenter of COVID-19 in the US at that time, 191,356 (155,726- 210,593) [72.9\% (59.3\%-80.0\%)] confirmed cases and $16,950(14,258-18,595)$ [77.9\% (65.5\%-85.5\%)] deaths would have been avoided if the same sequence of interventions had been applied one week earlier (Fig. 3C-D). A more pronounced control effect would have been achieved had the sequence of control measures occurred two weeks earlier: a reduction of 1,041,261 (996,933-1,076,703) [91.0\% (87.1\%94.0\%)] cases and 59,351 (56,238-61,789) [90.8\% (86.0\%94.5\%)] deaths in the US (Fig. 3E-F), and 254,087 (246,134257,738) [96.8\% (93.7\%-98.2\%)] cases and 21,175 (20,427$21,553)$ [97.3\% (93.9\%-99.0\%)] deaths in the New York metropolitan area (Fig. 3G-H). These dramatic reductions in morbidity and mortality due to more timely deployment of control measures highlight the critical need for aggressive, early response to the COVID-19 pandemic.

\section{Simulation of control relaxation and delayed response} Now that COVID-19 is established as a global pandemic, rapid response remains essential to avoid large-scale resurgences of infections and deaths in locations with reopening plans. We quantify the effect of response time on the timing and magnitude of rebound outbreaks in the US through further simulations. Specifically, we assume that control measures are relaxed beginning May 4, 2020 in all US counties, resulting in a weekly $5 \%$ increase in the local transmission rate, $\beta$, in each county. If weekly confirmed case numbers increase for 2 or 3 consecutive weeks in a county after relaxation, a reactive $25 \%$ weekly reduction of transmission rates, equivalent to the average transmission rate reduction prior to May 4, 2020 (Fig. S3), is imposed in this county and maintained until local weekly case numbers decline.

For both scenarios, a decline of daily confirmed cases continues for almost two weeks after easing of control measures (Fig. 4A-B). This decreasing trend, caused by the NPIs in place prior to May 4, 2020, coupled with the lag between infection acquisition and case confirmation, conveys a false signal that the pandemic is well under control. Unfortunately, due to high remaining population susceptibility, a large resurgence of cases follows, peaking in early- and mid-June, despite the resumption of NPI measures. For the 2-week response, increased mortality is less obvious due to a longer lag that disperses deaths over a longer time span (Fig. 4C, Fig. S7); however, a one-week further delay in local response to the resumption of control measures results in a marked resurgence in national deaths (Fig. 4D). Another scenario assuming a one-time $5 \%$ increase of transmission rates after control relaxation yields similar results.

Aggregating case and death numbers to national scale could mask drastic differences in local transmission. We inspected the relaxation simulations in six counties within the focus metropolitan areas (Fig. S8). For counties that have $R_{e}$ well below 1 (viz New York County NY, Orleans Parish LA and Suffolk County MA), relaxing control measures does not lead to increased cases and deaths, as the increased effective reproductive numbers remain below one. In contrast, reopening in counties with $R_{e}$ close to one (viz Los Angeles County CA, Cook County IL and Palm Beach County FL) do produce case growth.

To further highlight the effect of heterogeneous timelines of reopening among different locations, we also ran a simulation in which Florida reopens on May 4, 2020, resulting in a $20 \%$ increase in local transmission rates, but control measures in other states remain in place. We examined the daily cases and deaths in the following 30 days in Georgia and Alabama, the two states adjacent to Florida, and compared these outcomes with a baseline scenario in which no state reopens. The results indicate that reopening in Florida leads to increased numbers of cases and deaths in Georgia and Alabama (Fig. S9); this increase manifests with a oneweek lag.

\section{Discussion}

Unlike a local model describing the transmission dynamics 
within a single, independent site, the metapopulation model developed here allows study of the effect of asynchronous interventions across different locations. The transmission of SARS-CoV-2 in the US is a complex dynamical process with rapid spatial progression modulated by local control efforts. In particular, interventions in one location affect transmission in other places by altering the external force of infection via importations. Without spatial structure, the inference system cannot properly capture the dynamical coupling of disease transmission across locations.

The counterfactual experiments presented here (Fig. 3) are based on idealized assumptions. In practice, initiating and implementing interventions earlier during an outbreak is complicated by factors such as general uncertainty, economic concerns, logistics and the administrative decision process. Public compliance with social distancing rules may also lag due to sub-optimal awareness of infection risk. We acknowledge that our counterfactual experiments have simplified these processes; however, we note that by the end of February 2020, a number of other countries, including South Korea and Italy, were already aggressively responding to the virus (26). Our findings indicate that had control measures and reductions of $R_{e}$ in the US been implemented at a similar time, just 1-2 weeks earlier, substantially fewer cases and deaths would have occurred before May 3. Further, given that more effective control of COVID-19 has been maintained to date in countries such as South Korea, New Zealand, Vietnam and Iceland, these cases and deaths could have been averted, not merely postponed.

Our model experiments also indicate that rapid detection of increasing case numbers and fast re-implementation of control measures is needed to control rebound outbreaks of COVID-19 (Fig. 4). In these experiments, we assume the ability to re-implement a $25 \%$ weekly reduction of transmission rates nationwide. Due to general public fatigue toward NPIs and inconsistent compliance with control measures, this assumed reduction may be overly optimistic.

In this study, we have quantified the sensitivity of COVID19 cases and deaths to the timing of control measures. Our results demonstrate the dramatic impact that earlier interventions could have had on the COVID-19 pandemic in the US. Looking forward, the findings underscore the need for continued vigilance when control measures are relaxed. We recognize the burdens imposed by protracted shutdowns; however, it is vital to balance the dual ambitions of renewing social and economic activity and avoiding a recrudescence.

Countries such as South Korea, Vietnam, New Zealand and Germany, have shown that such a balance may be achievable; the strategies adopted in these countries could be used to guide policies in the US and elsewhere. Specifically, broader testing and contact tracing capacity (27) are crucial to detect a rebound of COVID-19 before it is well underway
(28). Susceptibility to SARS-CoV-2 infection remains high throughout the US (Fig. S6) and can readily support an exponential growth of cases and deaths (29). In addition, potential short-lived immunity against SARS-CoV-2 could replenish the susceptible population (30). Given this situation, economic re-opening and loosening of NPI measures would be more safely effected in localities in which $R_{e}$ is well below 1 , daily confirmed cases are low, and abundant testing and contact tracing are available to aid isolation and quarantine measures.

Since the initial submission of this paper at the end of May, following a relaxation of intervention measures, the US has experienced a massive resurgence of infections primarily driven by activity in southern states. In addition, some of the countries that managed to better limit COVID-19 transmission through July 2020 also saw rebounds of infections in August and September. These experiences underscore the necessity of maintaining control measures until sound public health targets are achieved, so that gains in outbreak control are preserved and the cumulative case burden over the entire course of the pandemic is substantially lower than what would result with no NPIs in place.

\section{Materials and Methods}

\section{The metapopulation model}

We use a metapopulation SEIR model to simulate the transmission of COVID-19 among 3,142 US counties. In this model, we consider two types of movement: daily work commuting and random movement. Information on county-to-county work commuting is publicly available from the US Census Bureau (15). We further assume the number of random visitors between two counties is proportional to the average number of commuters between them (13). As population present in each county is different during daytime and nighttime, we model the transmission dynamics of COVID-19 separately for these two time periods.

We formulate the transmission as a discrete Markov process during both day and night times. Daytime transmission lasts for $d t_{1}$ days and the nighttime transmission $d t_{2}$ days ( $\left.d t_{1}+d t_{2}=1\right)$. Here, we assume daytime transmission lasts for 8 hours and nighttime transmission lasts for 16 hours, i.e., $d t_{1}=1 / 3$ day and $d t_{2}=2 / 3$ day. A model with daytime and nighttime transmission each lasting for 12 hours $\left(d t_{1}=1 / 2\right.$ day and $d t_{2}=1 / 2$ day) yielded similar results (Fig. S4). The transmission dynamics are depicted by Eqs. S1-S10 in the Supplementary Materials. In these equations, we define $S_{i j}$, $E_{i j}, I_{i j}^{r}, I_{i j}^{u}$ and $N_{i j}$ as the susceptible, exposed, reported infected, unreported infected and total population in the subpopulation commuting from county $j$ to county $i(i \leftarrow j)$. 
We also introduce the following model parameters: $\beta$ is the transmission rate of reported infections; $\mu$ is the relative transmissibility of unreported infections; $Z$ is the average latency period (from infection to contagiousness); $D$ is the average duration of contagiousness; $\alpha$ is the fraction of documented infections; $\theta$ is a multiplicative factor adjusting random movement. Note in this model we assume a separate transmission rate, $\mu \beta$, for undocumented infections, many of whom may experience few or no symptoms. Our previous study in China indicates that the undocumented infections are less contagious than documented infections (17). Additionally, we assume a nationwide uniform ascertainment rate $\alpha$, given that per capita test numbers across different states are generally the same order of magnitude (Fig. S7). We integrated Eqs. S1-S10 using a Poisson process to represent the stochasticity of the transmission process.

The transmission model generates daily confirmed cases and deaths for each county. To map infections to deaths, we used an age-stratified infection fatality rate (IFR) and computed the IFR for each county as a weighted average using demographic information on local age structure (31). To account for reporting delays, we mapped simulated documented infections to confirmed cases using a separate observational delay model. In this delay model, we account for the time interval between a person transitioning from latent to contagious (i.e., $E \rightarrow I_{i}^{r}$ ) and observational confirmation of that individual infection. To estimate this delay period, $T_{d}$, we examined a US line-list data record consisting of 2.67 million confirmed cases (32). Prior to May 3, 2020, the time-to-event distribution of the interval (in days) from symptom onset to case confirmation is well fit by a gamma distribution ( $a=2.6, b=4.9$, mean $=12.9$ days, Fig. S7). Consequently, we adopted a gamma distribution to model $T_{d}$, but added another 2.5 days to the mean periods ( $a b$ ), as symptom onset is estimated to lag the onset of contagiousness (17). Recent studies on viral dynamics also indicate that pre-symptomatic infection is common and infected people can become contagious two days prior to symptom onset (33). As a result, we adopted $T_{d}=15.4$ days $(a=2.6, b=5.9)$ in this study. Based on daily incidence and death data in the US, the national death curve has a 7-day lag compared with the incidence curve (Fig. S7). As a result, we used a gamma distribution with a mean of 22.4 days ( $a=2.6, b=8.6$ ) to represent the delay between a person transitioning from latent to contagious and death.

In order to represent variability in transmission rates through space and time, we introduced separate estimates for $\beta$ in the 311 US counties with 400 or more cumulative cases as of May 3, 2020. The remaining counties were classified into 16 groups (evenly distributed into a 4 by 4 grouping based on cumulative cases and population density), for which separate transmission rates were defined. In total, 327 transmission rates $\left(\beta_{i}\right)$ were introduced in the transmission model. Using the next generation matrix approach, we derived the local basic reproductive number, $R_{t}=\beta D[\alpha+(1-\alpha) \mu]$. The effective reproductive number in each metropolitan area is the population weighted average of $R_{e}$ in constituent counties.

\section{Data}

We used the 2011-2015 5-Year ACS Commuting Flows data from US census survey to prescribe the inter-county movement in the transmission model prior to March 15, 2020, before broad control measures were announced. The county-tocounty commuting data are publicly available from the US Census Bureau (15). We visualize the inter-county commuting in Fig. S1. After March 15, the census survey data are no longer representative due to changes of mobility behavior in response to control measures. Therefore, after March 15, 2020 , we use estimates of the reduction of inter-county visitors to points of interest (POI) (e.g., restaurants, stores, etc.) to inform the decline of inter-county movement on a countyby-county basis (16). For instance, if the number of intercounty visitors was reduced by $30 \%$ in a county on a given day relative to baseline estimates on March 15, 2020, the size of subpopulations traveling to this county would be reduced by $30 \%$ accordingly. These real-time mobility data are available between March 1, 2020 and June 7, 2020. For dates beyond June 7, 2020, we maintained the last known level of inter-county movement.

County-level daily confirmed cases and deaths were compiled by USAFACTS (19). Daily cases and deaths in the six metropolitan areas are shown in Fig. S1.

\section{Model calibration}

To derive an estimate of model parameters, we calibrated the transmission model against county-level incidence data reported from February 21, 2020 through May 12, 2020 and death data reported from February 21, 2020 through May 19, 2020. Specifically, we estimated model parameters using a sequential data assimilation technique. The metapopulation model is a high-dimensional system with 60,232 subpopulations. We therefore applied an efficient data assimilation algorithm - the Ensemble Adjustment Kalman Filter (EAKF) (18), which is applicable to high dimensional model structures, to infer model parameters. The EAKF has been successfully used to infer parameters for seasonal and pandemic influenza as well as other infectious diseases (34-40).

To improve the identifiability of this high-dimensional model, we further reduced the number of unknown parameters by fixing disease-related parameters $(Z, D$ and $\mu)$ and 
the mobility factor $(\theta)$. These parameters were estimated using the posterior distributions inferred from case data through March 13, 2020 (14). Specifically, we randomly drew these parameters from the posterior ensemble members: $Z=3.59$ (95\% CI: 3.28-3.99), $D=3.56$ (3.21-3.83), $\mu=0.64$ (0.56-0.70), and $\theta=0.15$ (0.12-0.17).

From February 21, 2020 through May 3, 2020, we performed EAKF inference each day using both case and death data to estimate the ascertainment rate $\alpha$ and transmission rates $\beta_{i}$. The prior for the ascertainment rate was drawn from a distribution with a median value $\alpha=0.080$ (95\% CI: 0.069-0.093), estimated in a previous study. The prior transmission rates were scaled based on local population density using the following relation: $\beta_{i}=\frac{0.8 \times \log _{10}\left(P D_{i}\right)}{\text { median }\left(\log _{10}(P D)\right)} \times \beta_{0}$. Here $P D_{i}$ is the population density in county $i$, median $\left(\log _{10}(P D)\right)$ is the median value of log-transformed population density among all counties, and $\beta$ is the transmission rate estimated before March 13, 2020 ( $\beta_{0}=0.95$, 95\% CI: 0.84-1.06). For $\beta$ shared by multiple counties, population density $P D_{i}$ is averaged over those counties. To account for reporting delays of confirmed cases and deaths, at each daily model update we integrated the model forward for 14 days using the prior model state, and used incidence 10 days ahead and deaths 14 days ahead to constrain current model variables and parameters (i.e., the modes of gamma distributions for delays). Given the large number of parameters in the model, the inference system may not be fully identifiable. To alleviate this issue, we imposed a $\pm 30 \%$ limit on the daily change of parameters $\alpha$ and $\beta_{i}$. This smoothing constraint is reasonable considering the continuity of human behavioral change. Sensitivity tests obtained similar results with $\pm 25 \%$ and $\pm 35 \%$ smoothing constraints. A full list of settings for model parameters and variables is presented in Table 2 .

In total, we performed 20 independent inference runs. The inference results reported in Fig. 1 were obtained from all posterior ensemble members. Implementation details and system initialization are reported in the next section.

We evaluate the goodness-of-fit at county level using percentage absolute error (PAE) and percentage error (PE). Specifically, we define $P A E=\sum_{t}\left|f i t_{t}-o b s_{t}\right| / \sum_{t} o b s_{t} \quad$ and $P E=\sum_{t}\left(f i t_{t}-o b s_{t}\right) / \sum_{t} o b s_{t}$, where fit is the mean posterior fitting to case or death number and $o b s_{t}$ is the reported case or death number on day $t$. The PAE and PE values for case and death in 100 counties are reported in Table S1.

\section{System initialization}

To initialize the model, we seeded exposed individuals $(E)$ and unreported infections $\left(I^{u}\right)$ in counties with at least one confirmed case before March 14, 2020. Unlike the situation in China, where the outbreak originated from a single city, importation to multiple locations in the US likely initiated community transmission. To reflect this potential ongoing community transmission before the reporting of the first local infection, for each county with confirmed cases before March 14, we randomly drew $E$ and $I^{u}$ from uniform distributions $[0,20 C]$ and $[0,18 C] 9$ days prior to the reporting date $\left(T_{0}\right)$ of the first case. Here $C$ is the total number of reported cases between day $T_{0}$ and $T_{0}+4$.

The rationale for this seeding strategy is as follows. If an average reporting delay of 9 days is assumed, we can estimate $I^{r}$ on day $T_{0}-9$ as $\frac{C}{5} \times D$, where $\frac{C}{5}$ is the average number of daily cases during the first five days with reported cases ( $T_{0}$ to $\left.T_{0}+4\right)$. If we use the upper bound of 5 days for $D, I^{r}$ is estimated as $C$, which is also an upper bound. We assume the mean $I^{u}$ on day $T_{0}-9$ is $9 C$, implying a reporting rate of $1 / 10=10 \%$. Drawing $I^{u}$ from $[0,18 C]$ leads to a broader prior range of the reporting rate. As both $I^{r}$ and $I^{u}$ were evolved from the exposed population $E$, we draw $E$ from the range $[0,20 C]$. This crude calculation provides a seeding range for US counties. During inference, this seeding can be adjusted up or down by the filter. The posterior model fittings capture observed outcomes well (Table S1).

\section{Metropolitan areas}

In this study, we report the transmission dynamics in six metropolitan areas with dense populations and abundant observations: New York, New Orleans, Los Angeles, Chicago, Boston and Miami. The counties in these metropolitan areas are:

1. New York: Kings County NY, Queens County NY, New York County NY, Bronx County NY, Richmond County NY, Westchester County NY, Bergen County NJ, Hudson County NJ, Passaic County NJ, Putnam County NY, Rockland County $\mathrm{NY}$

2. New Orleans: Jefferson Parish LA, Orleans Parish LA, St. John the Baptist Parish LA, St. Tammany Parish LA

3. Los Angeles: Los Angeles County CA, Orange County CA

4. Chicago: Cook County IL, DuPage County IL, Kane County IL, McHenry County IL, Will County IL

5. Boston: Norfolk County MA, Plymouth County MA, Suffolk County MA

6. Miami: Miami-Dade County FL, Broward County FL, Palm Beach County FL 


\section{Seroprevalence surveys}

The seroprevalence of antibodies to SARS-CoV-2 in several locations in the United States is available from the US CDC website $(24,25)$. Here we used seroprevalence data available for dates before May 3, 2020. Those sites are: 1). NYC: Manhattan, Bronx, Queens, Kings, Nassau. 2). WA: King, Snohomish, Pierce, Kitsap, Grays Harbor. 3). LA: statewide. 4). SFL (south Florida): Miami-Dade, Broward, Palm Beach, Martin. 5). PA: Bucks, Chester, Cumberland, Delaware, Lancaster, Montgomery, Philadelphia. 6). MO: statewide. 7). SF (San Francisco): Martin, Contra Costa, Alameda, Santa Clara, San Mateo, San Francisco. 8). UT: statewide. 9). CT: statewide.

\section{Further validation of inference results}

We compared our inferred $R_{t}$ values at the state level with estimated $R_{t}$ values reported at https://rt.live. The Pearson correlation coefficient is 0.76 , indicating a general agreement in trend. A recent study estimated that 108,689 (95\% CI: 1,023 to $14,182,310$ ) infections occurred in the US before March 12, 2020 (41). Our inference estimated 236,207 (95\% CI: 193,855 to 298,937 ) total infections in the US by that date, which is within the $\mathrm{CI}$ and in line with the magnitude of the best estimate from that study.

\section{REFERENCES AND NOTES}

1. World Health Organization, Coronavirus disease (COVID-2019) situation reports. https://www.who.int/emergencies/diseases/novel-coronavirus-

2019/situation-reports/ (accessed June 22 2020).

2. Centers for Disease Control and Prevention, https://www.cdc.gov/coronavirus/2019-ncov/index.html (accessed June 22 2020)

3. M. L. Holshue, C. DeBolt, S. Lindquist, K. H. Lofy, J. Wiesman, H. Bruce, C. Spitters, K. Ericson, S. Wilkerson, A. Tural, G. Diaz, A. Cohn, L. Fox, A. Patel, S. I. Gerber, L. Kim, S. Tong, X. Lu, S. Lindstrom, M. A. Pallansch, W. C. Weldon, H. M. Biggs, T. M. Uyeki, S. K. Pillai; Washington State 2019-nCoV Case Investigation Team, First case of 2019 novel coronavirus in the United States. N. Engl. J. Med. 382, 929936 (2020). doi:10.1056/NEJMoa2001191 Medline

4. The New York Times, Coronavirus in the U.S.: Latest Map and Case Count. https://www.nytimes.com/interactive/2020/us/coronavirus-us-cases.html (accessed June 22 2020).

5. S. Lai, N. W. Ruktanonchai, L. Zhou, O. Prosper, W. Luo, J. R. Floyd, A. Wesolowski, M. Santillana, C. Zhang, X. Du, H. Yu, A. J. Tatem, Effect of non-pharmaceutical interventions to contain COVID-19 in China. Nature 585, 410-413 (2020). 10.1038/s41586-020-2293-x Medline

6. M. Chinazzi, J. T. Davis, M. Ajelli, C. Gioannini, M. Litvinova, S. Merler, A. Pastore Y Piontti, K. Mu, L. Rossi, K. Sun, C. Viboud, X. Xiong, H. Yu, M. E. Halloran, I. M. Longini Jr., A. Vespignani, The effect of travel restrictions on the spread of the 2019 novel coronavirus (COVID-19) outbreak. Science 368, 395-400 (2020). doi:10.1126/science. aba9757 Medline

7. H. Tian, Y. Liu, Y. Li, C. H. Wu, B. Chen, M. U. G. Kraemer, B. Li, J. Cai, B. Xu, Q. Yang, B. Wang, P. Yang, Y. Cui, Y. Song, P. Zheng, Q. Wang, O. N. Bjornstad, R. Yang, B. T. Grenfell, O. G. Pybus, C. Dye, An investigation of transmission control measures during the first 50 days of the COVID-19 epidemic in China. Science 368, 638-642 (2020). doi:10.1126/science.abb6105 Medline

8. B. F. Maier, D. Brockmann, Effective containment explains subexponential growth in recent confirmed COVID-19 cases in China. Science 368, 742-746 (2020). doi:10.1126/science.abb4557 Medline
9. J. Zhang, M. Litvinova, Y. Liang, Y. Wang, W. Wang, S. Zhao, Q. Wu, S. Merler, C. Viboud, A. Vespignani, M. Ajelli, H. Yu, Changes in contact patterns shape the dynamics of the COVID-19 outbreak in China. Science 368, 1481-1486 (2020). doi:10.1126/science.abb8001 Medline

10. M. U. G. Kraemer, C. H. Yang, B. Gutierrez, C. H. Wu, B. Klein, D. M. Pigott, L. du Plessis, N. R. Faria, R. Li, W. P. Hanage, J. S. Brownstein, M. Layan, A. Vespignani, H. Tian, C. Dye, O. G. Pybus, S. V. Scarpino; Open COVID-19 Data Working Group, The effect of human mobility and control measures on the COVID-19 epidemic in China. Science 368, 493-497 (2020). doi:10.1126/science.abb4218 Medline

11. J. Dehning, J. Zierenberg, F. P. Spitzner, M. Wibral, J. P. Neto, M. Wilczek, V. Priesemann, Inferring change points in the spread of COVID-19 reveals the effectiveness of interventions. Science 369, eabb9789 (2020). doi:10.1126/science.abb9789 Medline

12. H. Salje, C. Tran Kiem, N. Lefrancq, N. Courtejoie, P. Bosetti, J. Paireau, A. Andronico, N. Hozé, J. Richet, C.-L. Dubost, Y. Le Strat, J. Lessler, D. Levy-Bruhl, A. Fontanet, L. Opatowski, P.-Y. Boelle, S. Cauchemez, Estimating the burden of SARS-CoV-2 in France. Science 369, 208-211 (2020). doi:10.1126/science.abc3517 Medline

13. S. Pei, S. Kandula, W. Yang, J. Shaman, Forecasting the spatial transmission of influenza in the United States. Proc. Natl. Acad. Sci. U.S.A. 115, 2752-2757 (2018). doi:10.1073/pnas.1708856115 Medline

14. S. Pei, J. Shaman, Initial Simulation of SARS-CoV2 Spread and Intervention Effects in the Continental US. medRxiv https://doi.org/10.1101/2020.03.21.20040303 (2020).

15. United States Census Bureau, 2011-2015 5-Year ACS Commuting Flows. https://www.census.gov/data/tables/2015/demo/metro-micro/commutingflows-2015 html (accessed May 32020 ).

16. SafeGraph, https://www.safegraph.com/ (accessed May 32020 ).

17. R. Li, S. Pei, B. Chen, Y. Song, T. Zhang, W. Yang, J. Shaman, Substantial undocumented infection facilitates the rapid dissemination of novel coronavirus (SARS-CoV-2). Science 368, 489-493 (2020). doi:10.1126/science.abb3221 Medline

18. J. L. Anderson, An ensemble adjustment Kalman filter for data assimilation. Mon $\begin{array}{llll}\text { Weather Rev. 129, 2884-2903 (2001). doi:10.1175/1520- } & \end{array}$ 0493(2001)129<2884:AEAKFF>2.0.C0:2

19. USAFACTS, https://usafacts.org/visualizations/coronavirus-covid-19-spreadmap/ (accessed May 4 2020).

20. O. Diekmann, J. A. P. Heesterbeek, M. G. Roberts, The construction of nextgeneration matrices for compartmental epidemic models. J. R. Soc. Interface 7 , 873-885 (2009). doi:10.1098/rsif.2009.0386 Medline

21. The COVID Analysis and Mapping of Policies Project, https://covidlocal.org/amp/ (accessed September 12020)

22. https://github.com/shaman-lab/Counterfactual.

23. J. D. Goodman, M. Rothfeld, 1 in 5 New Yorkers May Have Had Covid-19, Antibody Tests Suggest. https://www.nytimes.com/2020/04/23/nyregion/coronavirusantibodies-test-ny.html (accessed June 22 2020).

24. F. P. Havers, C. Reed, T. Lim, J. M. Montgomery, J. D. Klena, A. J. Hall, A. M. Fry, D. L. Cannon, C.-F. Chiang, A. Gibbons, I. Krapiunaya, M. Morales-Betoulle, K. Roguski, M. A. U. Rasheed, B. Freeman, S. Lester, L. Mills, D. S. Carroll, S. M. Owen, J. A. Johnson, V. Semenova, C. Blackmore, D. Blog, S. J. Chai, A. Dunn, J. Hand, S. Jain, S. Lindquist, R. Lynfield, S. Pritchard, T. Sokol, L. Sosa, G. Turabelidze, S. M. Watkins, J. Wiesman, R. W. Williams, S. Yendell, J. Schiffer, N. J. Thornburg, Seroprevalence of Antibodies to SARS-CoV-2 in 10 Sites in the United States, March 23-May 12, 2020. JAMA Intern. Med. (2020), doi:10.1001/iamainternmed.2020.4130

25. Large-scale Geographic Seroprevalence Surveys, https://www.cdc.gov/coronavirus/2019-ncov/cases-updates/geographicseroprevalence-surveys.html (accessed September 12020).

26. S. Hsiang, D. Allen, S. Annan-Phan, K. Bell, I. Bolliger, T. Chong, H. Druckenmiller, L. Y. Huang, A. Hultgren, E. Krasovich, P. Lau, J. Lee, E. Rolf, J. Tseng, T. Wu, The effect of large-scale anti-contagion policies on the COVID-19 pandemic. Nature 584, 262-267 (2020). doi:10.1038/s41586-020-2404-8 Medline

27. L. Ferretti, C. Wymant, M. Kendall, L. Zhao, A. Nurtay, L. Abeler-Dörner, M. Parker D. Bonsall, C. Fraser, Quantifying SARS-CoV-2 transmission suggests epidemic control with digital contact tracing. Science 368, eabb6936 (2020). 
doi:10.1126/science.abb6936 Medline

28. T. Yamana, S. Pei, J. Shaman, Projection of COVID-19 Cases and Deaths in the U as Individual States Re-open May 4,2020. medRxiv https://doi.org/10.1101/2020.05.04.20090670 (2020).

29. R. E. Baker, W. Yang, G. A. Vecchi, C. J. E. Metcalf, B. T. Grenfell, Susceptible supply limits the role of climate in the early SARS-CoV-2 pandemic. Science 369, 315319 (2020). Medline

30. S. M. Kissler, C. Tedijanto, E. Goldstein, Y. H. Grad, M. Lipsitch, Projecting the transmission dynamics of SARS-CoV-2 through the postpandemic period. Science 368, 860-868 (2020). doi:10.1126/science.abb5793 Medline

31. R. Verity, L. C. Okell, I. Dorigatti, P. Winskill, C. Whittaker, N. Imai, G. CuomoDannenburg, H. Thompson, P. G. T. Walker, H. Fu, A. Dighe, J. T. Griffin, M. Baguelin, S. Bhatia, A. Boonyasiri, A. Cori, Z. Cucunubá, R. FitzJohn, K. Gaythorpe, W. Green, A. Hamlet, W. Hinsley, D. Laydon, G. Nedjati-Gilani, S. Riley, S. van Elsland, E. Volz, H. Wang, Y. Wang, X. Xi, C. A. Donnelly, A. C. Ghani, N. M. Ferguson, Estimates of the severity of coronavirus disease 2019: A model-based analysis. Lancet Infect. Dis. 20, 669-677 (2020). doi:10.1016/S14733099(20)30243-7 Medline

32. COVID-19 Case Surveillance Public Use Data, https://data.cdc.gov/CaseSurveillance/COVID-19-Case-Surveillance-Public-Use-Data/vbim-akgf (accessed August 282020).

33. X. He, E. H. Y. Lau, P. Wu, X. Deng, J. Wang, X. Hao, Y. C. Lau, J. Y. Wong, Y. Guan, X. Tan, X. Mo, Y. Chen, B. Liao, W. Chen, F. Hu, Q. Zhang, M. Zhong, Y. Wu, L. Zhao, F. Zhang, B. J. Cowling, F. Li, G. M. Leung, Temporal dynamics in viral shedding and transmissibility of COVID-19. Nat. Med. 26, 672-675 (2020). doi:10.1038/s41591-020-0869-5 Medline

34. W. Yang, M. Lipsitch, J. Shaman, Inference of seasonal and pandemic influenza transmission dynamics. Proc. Natl. Acad. Sci. U.S.A. 112, 2723-2728 (2015). doi:10.1073/pnas.1415012112 Medline

35. S. Pei, F. Morone, F. Liljeros, H. Makse, J. L. Shaman, Inference and control of the nosocomial transmission of methicillin-resistant Staphylococcus aureus. eLife 7 , e40977 (2018). doi:10.7554/eLife.40977 Medline

36. J. Reis, J. Shaman, Retrospective parameter estimation and forecast of respiratory syncytial virus in the United States. PLOS Comput. Biol. 12, e1005133 (2016). doi:10.1371/journal.pcbi.1005133 Medline

37. A. K. Heaney, K. A. Alexander, J. Shaman, Ensemble forecast and parameter inference of childhood diarrhea in Chobe District, Botswana. Epidemics 30 , 100372 (2020). doi:10.1016/i.epidem.2019.100372 Medline

38. J. Shaman, A. Karspeck, Forecasting seasonal outbreaks of influenza. Proc. Natl. Acad. Sci. U.S.A. 109, 20425-20430 (2012). doi:10.1073/pnas.1208772109 Medline

39. N. B. DeFelice, E. Little, S. R. Campbell, J. Shaman, Ensemble forecast of human West Nile virus cases and mosquito infection rates. Nat. Commun. 8, 14592 (2017). doi:10.1038/ncomms14592 Medline

40. S. Pei, J. Shaman, Counteracting structural errors in ensemble forecast of influenza outbreaks. Nat. Commun. 8, 925 (2017). doi:10.1038/s41467-01701033-1 Medline

41. T. A. Perkins, S. M. Cavany, S. M. Moore, R. J. Oidtman, A. Lerch, M. Poterek, Estimating unobserved SARS-CoV-2 infections in the United States. Proc. Natl. Acad. Sci. U.S.A. 117, 22597-22602 (2020). doi:10.1073/pnas.2005476117 Medline

\section{ACKNOWLEDGMENTS}

We thank SafeGraph for sharing the human mobility data and Columbia University Mailman School of Public Health for high-performance computing resources. Funding: This study was supported by funding from the National Institutes of Health (GM110748) and the National Science Foundation (DMS-2027369), as well as a gift from the Morris-Singer Foundation. Author contributions: S.P. and J.S. designed the study. S.P. and S.K. performed the analysis. All authors wrote and reviewed the manuscript. Competing interests: J.S. and Columbia University disclose partial ownership of SK Analytics. J.S. discloses consulting for BNI. All other authors declare no competing interests. Data and materials availability: All data needed to evaluate the conclusions in the paper are present in the paper and/or the Supplementary Materials. Code and data are available at https:/github.com/shaman-lab/Counterfac-

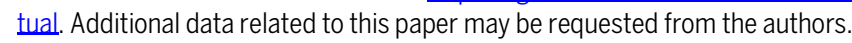

\section{SUPPLEMENTARY MATERIALS}

advances.sciencemag.org/cgi/content/full/sciadv.abd6370/DC1

Submitted 2 July 2020

Accepted 20 October 2020

Published First Release 6 November 2020

$10.1126 /$ sciadv.abd6370 

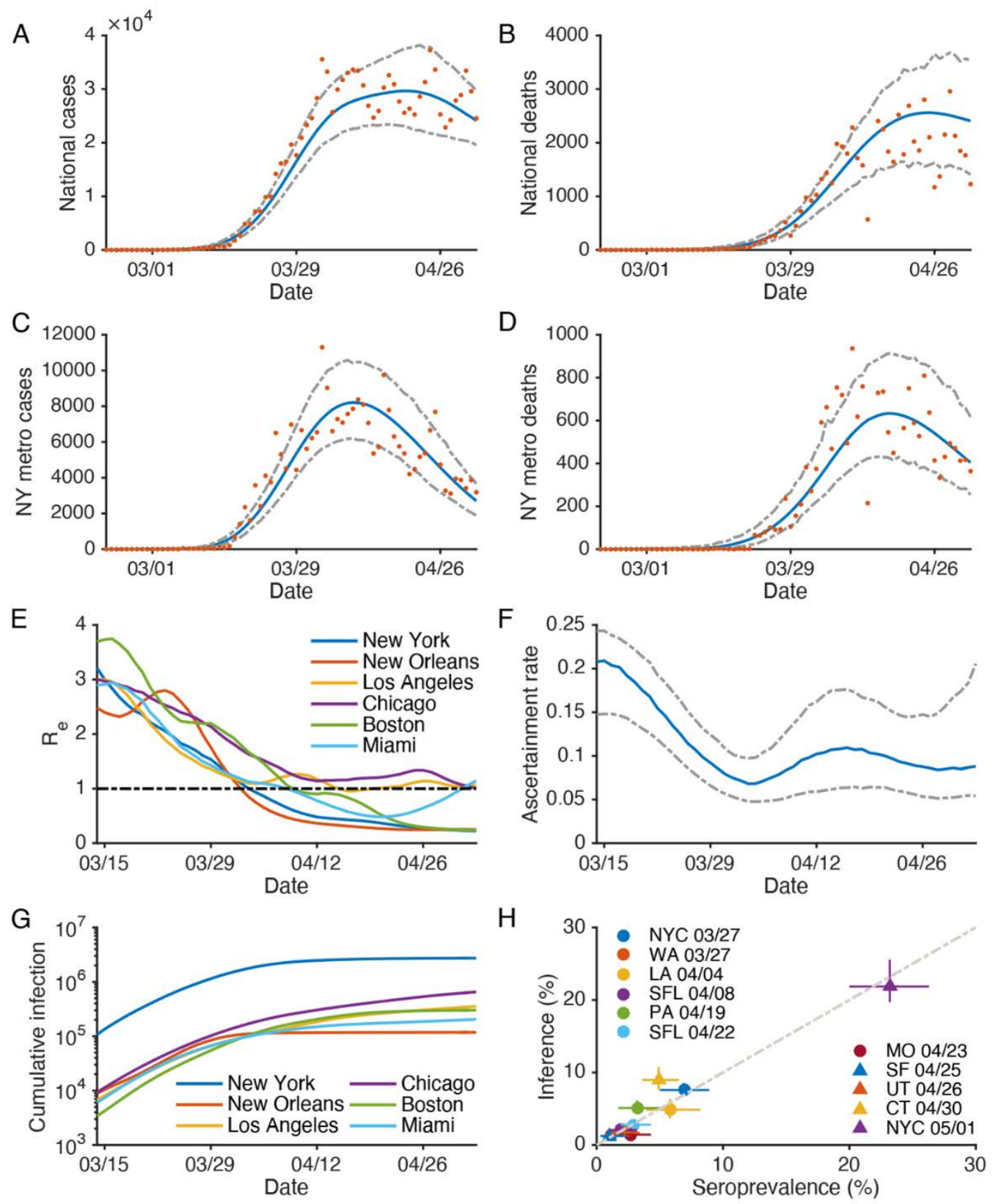

Fig. 1. Model fit and parameter inference. Posterior fitting to daily cases and deaths in the US (A-B) and the New York metropolitan area (C-D). Red dots represent observations. Blue and grey lines are the median estimate and $95 \% \mathrm{Cls}$. The estimated effective reproductive number, $R_{e}$, in six metropolitan areas are shown in (E). The black dotted line indicates $R_{e}=1$ . Panel $(F)$ shows the estimated ascertainment rate over time. The blue line and grey lines are the median estimates and $95 \% \mathrm{Cls}$. Panel (G) shows the estimated cumulative infections (both reported and unreported) in six metropolitan areas. We compare the reported seroprevalence (\%) in nine locations on different dates with the inferred percentage cumulative infections on those dates in $(\mathrm{H})$. Whiskers show $95 \% \mathrm{Cls}$. Details on the serological survey are provided in Materials and Methods. 

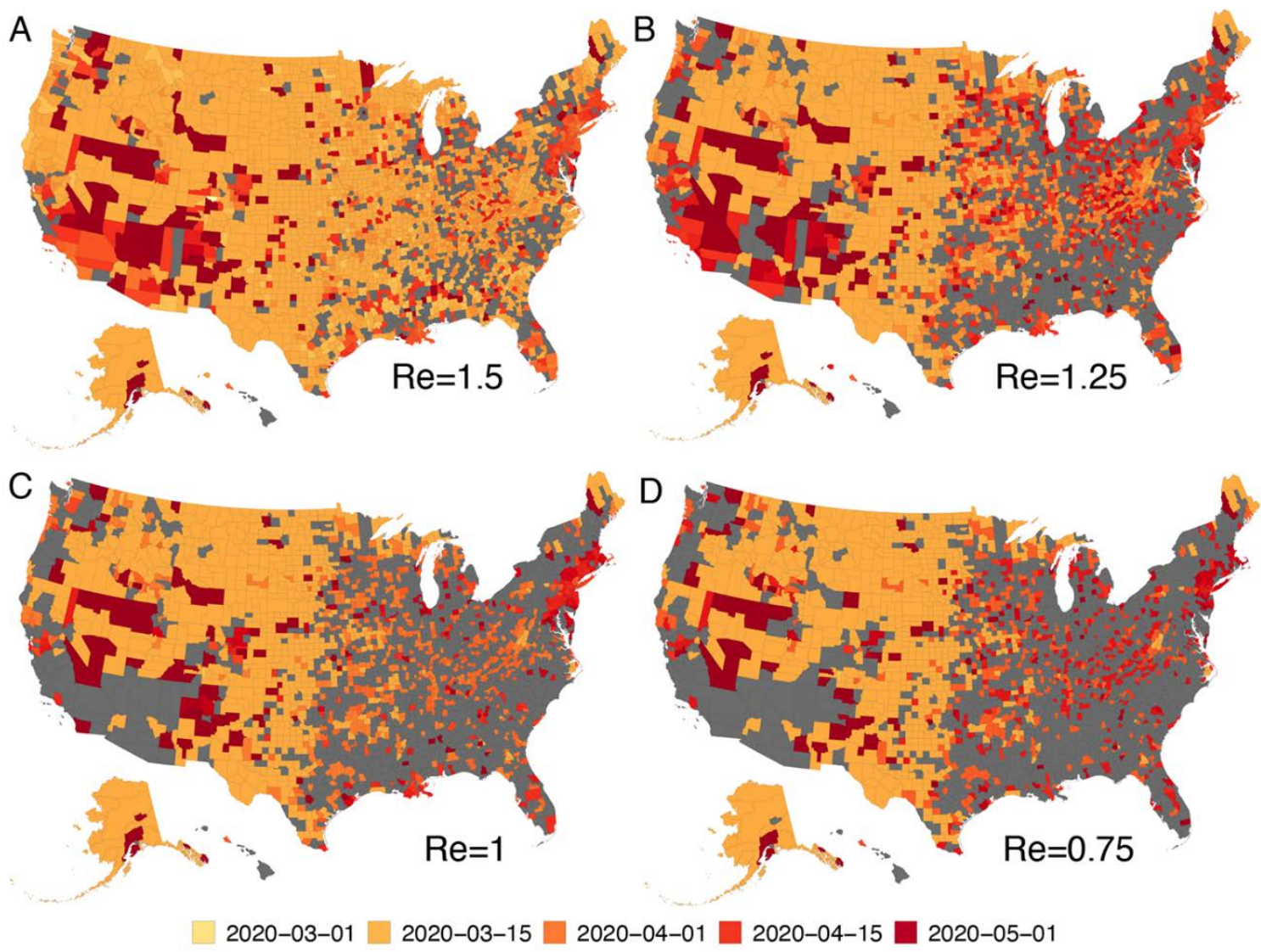

Fig. 2. Asynchronous reduction of effective reproductive numbers. For each county, we show the date when the local effective reproductive number dropped below $1.5(A), 1.25$ (B), 1 (C) and 0.75 (D), and maintained below that threshold until May 3. Counties in grey are those that either never reached the threshold or failed to remain below the threshold. 

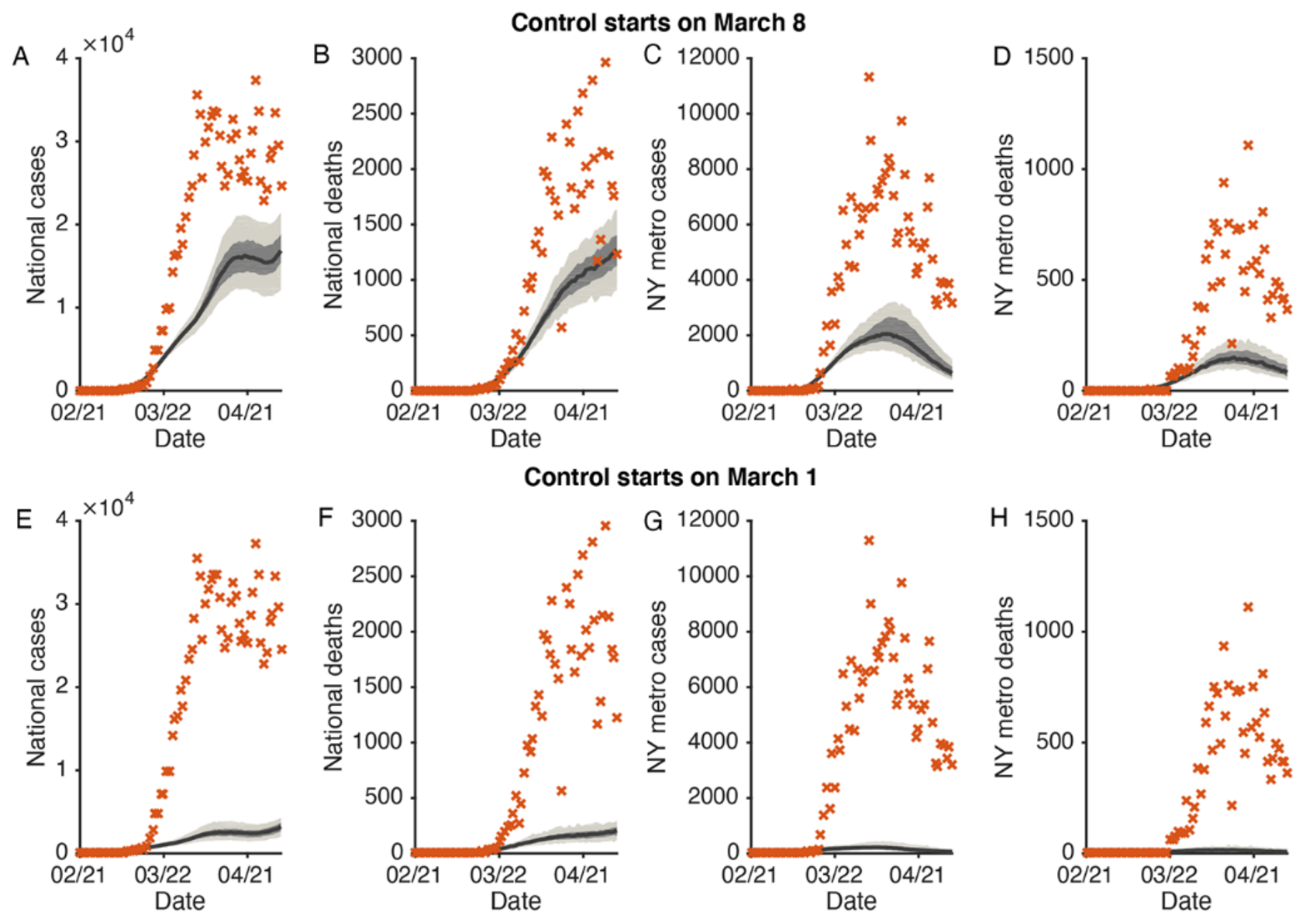

Control starts on March 1
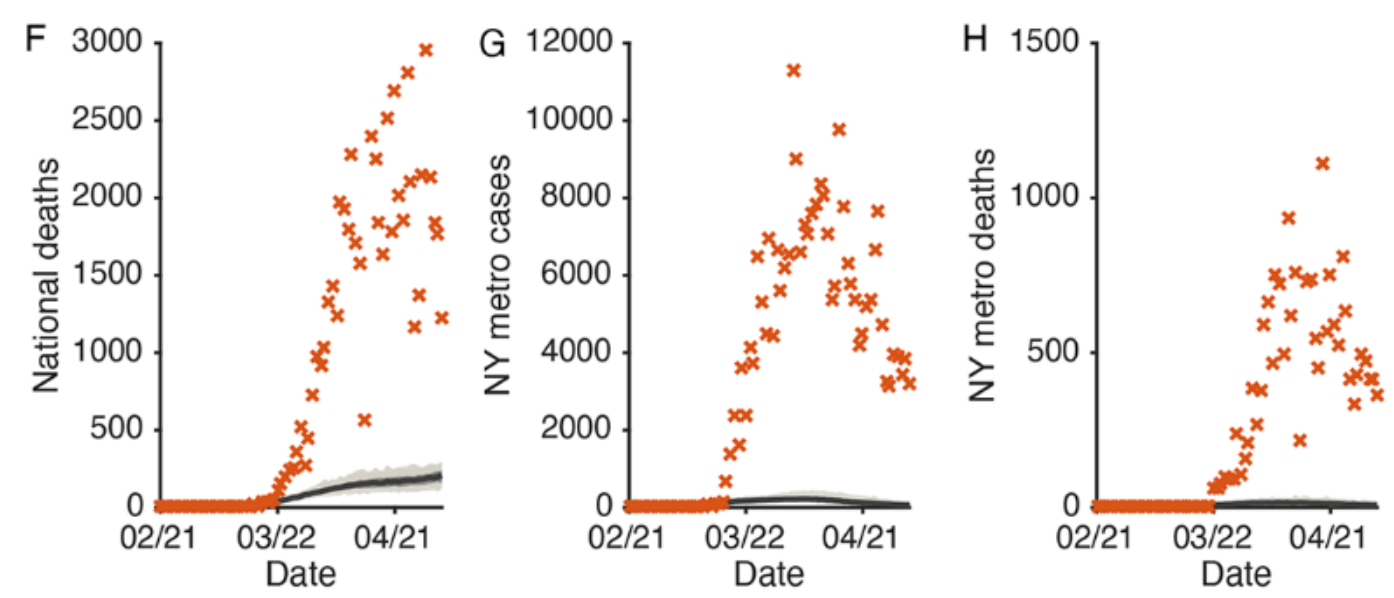

Fig. 3. Counterfactual simulations with control interventions beginning in early March -1 and 2 weeks earlier than implemented. Daily cases and deaths in the US (A, B, E, F) and the New York metropolitan area $(C, D, G, H)$ under early interventions are compared with the observations (red crosses). The upper and lower rows present counterfactuals with interventions implemented on March 8 and March 1, respectively. The black lines and surrounding bands show the median estimate, interquartile and $95 \% \mathrm{Cls}$. 

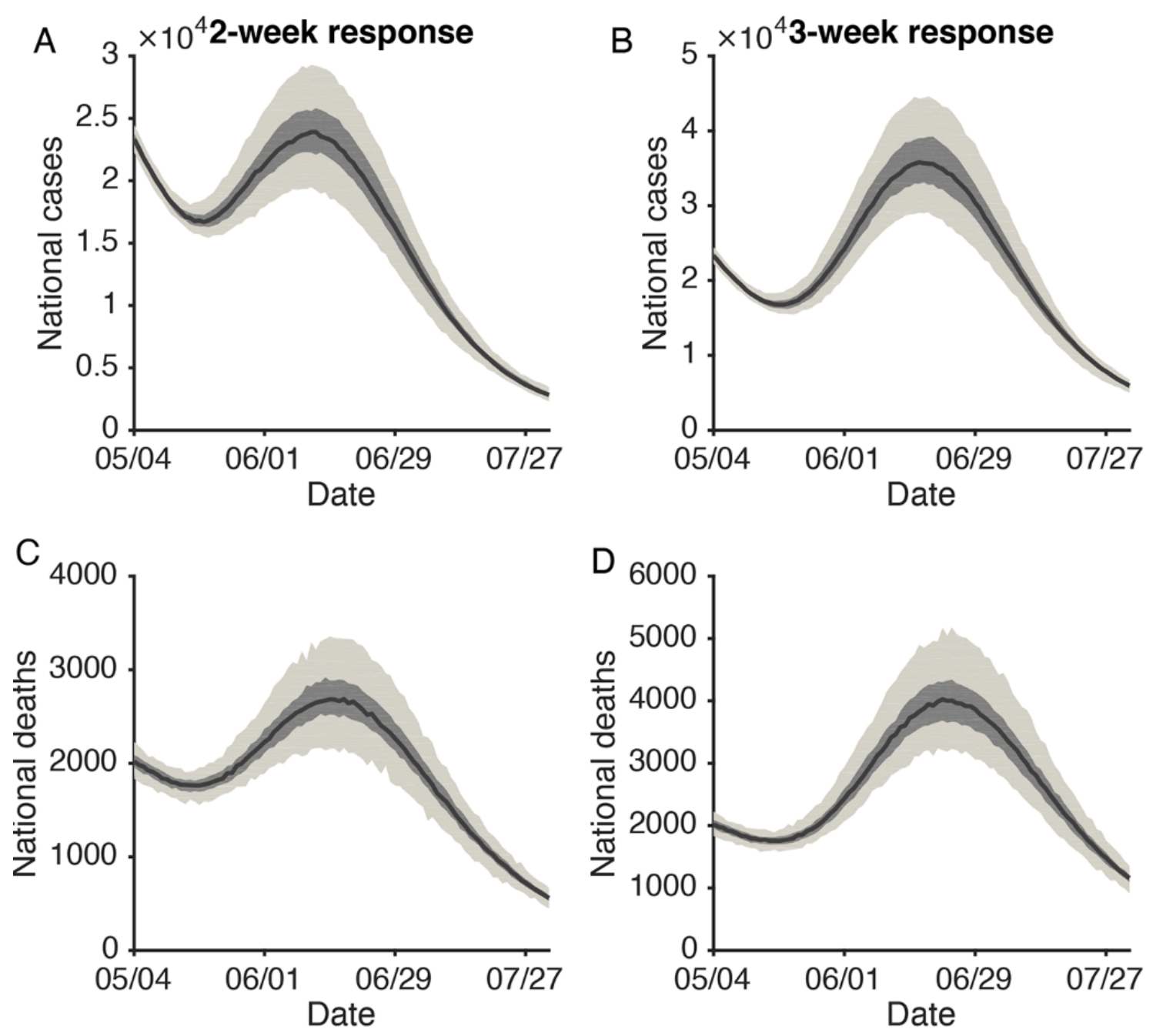

Fig. 4. Effects of response time after control measures are relaxed. We assume a control relaxation (a weekly $5 \%$ increase of the transmission rate) starting on May 4 in all US counties. If the local weekly case number in a county increases for 2 or 3 consecutive weeks, a weekly $25 \%$ reduction of the transmission rate is imposed for that county. Daily cases and deaths in the US for a response time of 2 weeks (A, C) and 3 weeks (B, D) are compared. The black lines and bands show the median estimate, interquartile and $95 \% \mathrm{Cls}$. 
Table 1. Estimated basic reproductive numbers. Estimated basic reproductive numbers $\left(R_{t}\right)$ for the New York, New Orleans, Los Angeles, Chicago, Boston and Miami metropolitan areas on March 15, March 29, April 12, April 26 and May 3. Mean estimate (95\% CIs) are presented.

\begin{tabular}{clllll}
\hline & March 15 & March 29 & April 12 & April 26 & May 3 \\
\hline \multirow{2}{*}{ New York } & 3.10 & 1.63 & 0.60 & 0.38 & 0.34 \\
& $(2.76,3.45)$ & $(1.41,1.88)$ & $(0.52,0.66)$ & $(0.32,0.43)$ & $(0.30,0.40)$ \\
New Orleans & 2.50 & 1.91 & 0.44 & 0.29 & 0.27 \\
& $(2.11,2.89)$ & $(1.65,2.27)$ & $(0.34,0.55)$ & $(0.22,0.36)$ & $(0.21,0.34)$ \\
Los Angeles & 3.12 & 1.35 & 1.16 & 1.17 & 1.18 \\
& $(2.56,3.72)$ & $(1.06,1.71)$ & $(0.89,1.38)$ & $(0.89,1.44)$ & $(0.91,1.44)$ \\
Chicago & 2.84 & 2.04 & 1.11 & 1.39 & 1.13 \\
& $(2.41,3.34)$ & $(1.74,2.36)$ & $(0.92,1.29)$ & $(1.14,1.66)$ & $(0.92,1.34)$ \\
Boston & 3.86 & 2.19 & 0.95 & 0.41 & 0.33 \\
& $(3.28,4.47)$ & $(1.73,2.57)$ & $(0.77,1.08)$ & $(0.32,0.54)$ & $(0.26,0.40)$ \\
Miami & 2.96 & 1.38 & 0.78 & 0.76 & 1.32 \\
& $(2.56,3.45)$ & $(1.12,1.68)$ & $(0.60,0.96)$ & $(0.57,0.88)$ & $(1.07,1.52)$ \\
\hline
\end{tabular}


Table 2. Setting initial parameters and variables. The prior transmission rate in each county is scaled by population density using a baseline transmission rate $\beta_{0}$ as inferred through March 13, 2020. The relative transmission rate ( $\mu$ ), latency period ( $Z$ ), infectious period $(D)$ and mobility factor $(\theta)$ are fixed at posterior values inferred through March 13, 2020. Values are shown for the median and 95\% CIs in the parentheses. The initial numbers of exposed individuals $E$ and unreported infected individuals $I^{u}$ are drawn from uniform distributions $U(0,18 C)$ and $U(0,20 C) 9$ days prior to the reporting date $\left(T_{0}\right)$ of the first case. Here $C$ is the total number of reported cases between days $T_{0}$ and $T_{0}+4$.

\begin{tabular}{ll}
\hline Parameter/variable & Values \\
\hline Baseline transmission rate $\left(\beta_{0}\right.$, day $\left.^{-1}\right)$ & $0.95(0.84,1.06)$ \\
Relative transmission rate $(\mu)$ & $0.64(0.56,0.70)$ \\
Latency period $(Z$, day) & $3.59(3.28,3.99)$ \\
Infectious period $(D$, day) & $3.56(3.21,3.83)$ \\
Mobility factor $(\theta)$ & $0.15(0.12,0.17)$ \\
Reporting rate $(\alpha)$, prior & $0.080(0.069,0.093)$ \\
Initial exposed population $(E)$ & $U(0,20 C)$ \\
Initial unreported infected population $\left(I^{u}\right)$ & $U(0,18 C)$ \\
\hline
\end{tabular}

